\title{
LA GEOMETRÍA ANALÍTICA EN EL CURSO COMPLETO DE MATEMÁTICAS PURAS (1829) DE JOSÉ DE ODRIOZOLA
}

\author{
The analytical geometry in the Curso completo de matemáticas \\ puras (1829) by José de Odriozola
}

\section{Isabel M. Sánchez Sierra ${ }^{\alpha}$ y María Teresa González Astudilloo}

Fecha de recepción: 03/14/2019 • Fecha de aceptación: 16/05/2019

Resumen. La enseñanza de la Geometría Analítica en España en los inicios del siglo XIX muestra ciertas reminiscencias de la Geometría de Descartes, aunque también incorpora el uso de sistemas de coordenadas casi como lo hacemos en la actualidad. La obra Curso Completo de Matemáticas puras escrita entre 1827 y 1829 por José Odriozola es una buena muestra de ello. De hecho, en el libro encontramos estas dos formas de hacer Geometría Analítica, aunque claramente diferenciadas en apartados distintos. En este artículo se hace una breve semblanza del autor, se sitúa en el contexto social y educativo de la época y se muestran los contenidos que formaban parte de cada uno de los dos apartados indicados. En cuanto al primero, se señala la forma en que eran construidas geométricamente las expresiones algebraicas, la interpretación geométrica que se les daba, los problemas que se planteaban, la interpretación de las soluciones negativas de dichos problemas y las limitaciones de las expresiones algebraicas a ecuaciones homogéneas. Estos dos últimos aspectos fueron un caballo de batalla para todos los autores de libros de texto, al menos de la primera mitad del siglo XIX. Así mismo, se presentan los sistemas de coordenadas considerados en el libro y la utilidad que se les daba para resolver algunos problemas geométricos.

Palabras-clave: Geometría Analítica; Libros de texto; Historia de la educación.

\footnotetext{
${ }^{\alpha}$ Departamento de matemáticas. IES Francisco de Salinas. Salamanca. c/Julita Ramos, s/n. 37004 Salamanca. España. isamss@yahoo.com (D) https://orcid.org/0000-0003-1396-7408

B Departamento de Didáctica de la Matemática y Didáctica de las Ciencias Experimentales. Facultad de Educación. Universidad de Salamanca. Paseo de Canalejas, 169. 37008 Salamanca. España. maite@usal.es (D) http://orcid.org/0000-0003-4800-365X
} 
Abstract. While the teaching of analytical geometry in Spain at the beginning of the 19th century shows some reminiscences of the geometry of Descartes, it also incorporates the use of coordinate systems, much as is done today. The work Curso Completo de Matemáticas Puras, by José Odriozola, published between 1827 and 1829, is a good example of this. In fact, in this book we find both ways of doing analytical geometry, although they are clearly differentiated into different sections.

In this article we provide a brief biography of Odriozola, an overview of the socio-educative context of the time, and a look at the contents that were part of each of the two sections mentioned. The first section - that of analytic geometry - describes how algebraic expressions were constructed, the geometric interpretations that were given, the problems raised, the interpretation of negative solutions to these problems and the limitations of the algebraic expressions to homogeneous equations. These last two aspects were a bone of contention for every textbook author, at least during the first half of the nineteenth century. Likewise, we analyze the coordinate systems that appear in the book, along with the utility that was given to these systems for solving certain geometric problems.

Keywords: Analytical geometry; Textbooks; History of education.

\section{INTRODUCCIÓN}

La Geometría Analítica nace en el siglo XVII de la mano de René Descartes (1596-1650) y Pierre Fermat (1601-1665). En un principio será Descartes quién pase a la historia como el padre de la misma gracias a su Geometría (1637), apéndice del Discurso del método, y ello a pesar de que la geometría que describe en esta obra se parece poco a lo que hoy consideramos como Geometría Analítica. Actualmente también se reconoce a Fermat dicha autoría pues, su obra Ad locos planos et solidos isagoge, publicada póstumamente en 1679, fue escrita antes de la aparición de La Geometría.

En la obra de Odriozola, y en general en todas las obras de Geometría Analítica utilizadas en España en la primera mitad del siglo XIX, encontramos una Geometría Analítica muy próxima a los métodos de Descartes, por lo que a continuación describiremos estos brevemente antes de llevar a cabo el análisis del libro que nos ocupa.

La notación utilizada por Descartes en su Geometría es muy similar a la actual, pero desde el punto de vista conceptual existe una diferencia 
muy importante entre ambas: mientras que ahora consideramos a los parámetros y a las incógnitas como cantidades, Descartes las considera como segmentos. Esto plantea dos problemas. El primero de ellos es que mientras que con las letras pueden realizarse operaciones aritméticas en número ilimitado, con los segmentos tales combinaciones quedan reducidas al caso en que el grado de la expresión resultante es uno, dos o tres, pues en los otros casos ese resultado deja de ser expresable en términos de figuras geométricas. Por otra parte, las ecuaciones obtenidas deben ser homogéneas, pues no tiene sentido sumar un «área» $\left(\mathrm{x}^{2}\right)$, con un «volumen» $\left(\mathrm{x}^{3}\right)$, por ejemplo. Para superar tal limitación Descartes recurre a la idea del segmento unitario: un segmento arbitrario adoptado como unidad y que, operando con él convenientemente, reduce toda combinación de segmentos, cualquiera que sea su dimensión, a una expresión homogénea. Por otra parte, esa unidad irá sobrentendida y, de hecho, ni ella ni sus operaciones serán explícitas. Así, para operar con segmentos simplemente es necesario indicar con una letra cada uno de los datos, y el resultado como la combinación respectiva de esas letras de acuerdo con las reglas del álgebra. Además, Descartes muestra cómo se interpretan geométricamente las operaciones algebraicas y aplica todo ello a la resolución de problemas geométricos. Para ello traduce el problema al lenguaje algebraico utilizando la equivalencia entre segmentos y letras que acabamos de describir, resuelve algebraicamente la ecuación obtenida y finalmente construye con regla y compás la expresión algebraica correspondiente a la solución. ${ }^{1}$

Como hemos dicho anteriormente, encontraremos una manera análoga de hacer Geometría Analítica en la obra de Odriozola que mostramos en este trabajo.

El segundo problema que surge con esta manera de aplicar el Álgebra a la Geometría es la interpretación y el manejo de las soluciones negativas, que representan segmentos, y que Descartes desecha por falsas. Sin embargo, los matemáticos del siglo XIX buscarán una interpretación de las mismas en el contexto del problema.

El objetivo de este trabajo es analizar la forma en la que Odriozola plantea la enseñanza de la Geometría Analítica en cuanto al tratamiento

\footnotetext{
1 René Descartes, La Geometría (Buenos Aires: Espasa-Calpe, 1947 [1637]). Traducción de Pedro Rosell. David Eugene Smith y Marcia L. Latham, The Geometry of René Descartes: Translated from de French and Latin, with a facsimil of the first edition, 1637 (Illinois: Open Court, 1952).
} 
que da a las expresiones algebraicas y a las soluciones negativas de los problemas, así como al uso de los sistemas de coordenadas.

\section{EL AUTOR}

José Odriozola fue un matemático, militar y artista español nacido en Cestona (Guipúzcoa) (1785-1864). Sus primeros estudios los realizó en la Real Academia de San Fernando (Cádiz), de la que sería nombrado académico de mérito en 1814. En su estancia en San Fernando adquirió conocimientos matemáticos, ya que el centro cuidaba mucho estas enseñanzas. ${ }^{2}$

En 1808 se incorporó como cadete a los voluntarios de Borbón, en Galicia, comenzando así su carrera militar, llegando a ser coronel de Infantería y teniente coronel de Artillería. ${ }^{3}$ En 1810 consiguió su primer cargo docente, como profesor de batallón de cadetes, en Andalucía. Tras esto ocupó los cargos de profesor ayudante en los colegios de Artillería de Sevilla (1813), Segovia (1814), Badajoz (1823) y Alcalá de Henares (1830). Entre 1823, año en que cerró el Colegio de Badajoz, y 1830 en que se incorporó al de Alcalá, Odriozola preparó algunos de sus tratados técnicos y científicos destinados a centros superiores de formación militar. Entre ellos se encuentra el Curso Completo de Matemáticas Puras que se publicó entre 1827 y 1829, escrito por encargo de la Junta Superior Facultativa del Real Cuerpo de Artillería, ${ }^{4}$ aunque posteriormente se utilizaría en la segunda enseñanza.

Durante 1834-35 realizó un viaje por Europa, donde se familiarizó con las industrias y técnicas militares más avanzadas, primero en Francia y Alemania y después en Inglaterra. En este viaje Odriozola adquirió una predilección por el saber técnico frente al científico, como base del desarrollo del país y durante la década siguiente intentó poner en práctica estas ideas, sobre todo en el Seminario de Bergara, del que fue nombrado director en 1845. ${ }^{5}$ En 1840 escribió unas «Normas para el fomento de la enseñanza

\footnotetext{
2 Enciclopedia Universal Ilustrada Europeo-Americana, «Odriozola» (Madrid: Espasa-Calpe, S.A., 1929), t. 39, 745.

3 Alexander Maz, «Los números negativos en España en los siglos XVIII y XIX» (Tesis doctoral, Universidad de Granada, 2005), 298-299.

${ }^{4}$ Enciclopedia Auñamendi, Fondo Bernardo Estornés Lasa, «Odriozola Oñativia, José», Enciclopedia Auñamendi. Fondo Bernardo Estornés Lasa. http://www.euskomedia.org/aunamendi/99088 (consultado en julio de 2014).
}

${ }_{5}^{5}$ Enciclopedia Auñamendi, «Odriozola Oñativia, José». 
primaria y secundaria» en las que defendía las asignaturas científicas con una clara orientación práctica y experimental. Vea opina que este autor fue «uno de los pocos matemáticos españoles de la primera mitad del XIX con iniciativa propia para mejorar la situación académica de las matemáticas, tanto en la enseñanza militar como en su posterior repercusión en la enseñanza tanto elemental como de ampliación». ${ }^{6}$

Otro de los indicios que muestran el interés de Odriozola por el desarrollo de la ciencia fue su participación en la fundación de la Real Academia de Ciencias Exactas, Físicas y Naturales en 1847.

Este autor publicó numerosos tratados científicos entre los que citaremos: Tratado elemental de Mecánica (1832), Mecánica Aplicada a las máquinas operando, o Tratado teórico y experimental sobre el trabajo de las fuerzas (1839) y Curso Completo de Matemáticas Puras (1827-1829), que es la obra que nos ocupa.

\section{LA OBRA $^{7}$}

Esta obra fue escrita para su uso en las academias militares, como se desprende de la carta, incluida al comienzo del tomo I, publicado en 1827, dirigida a D. Miguel de Ibarrola, Secretario del Estado y del despacho Universal de la Guerra, en la que le presenta el libro, indicando además que ha "procurado esplicar las materias con la estension propia de su estado actual en Europa».8 Pero posteriormente se recomendó como texto para la segunda enseñanza, apareciendo en las listas oficiales de libros aprobados por el gobierno para el curso 1846-47, recogidas en la Gaceta de Madrid de 8 de septiembre de 1846 .

Así mismo, en el prólogo Odriozola especifica el nivel al que va destinado, indicando «que un curso completo de Matemáticas no es obra para escuelas de primera enseñanza». ${ }^{9}$ Añade también:

\footnotetext{
- Fernando Vea, Las matemáticas en la enseñanza secundaria en España en el siglo XIX (Zaragoza: Seminario de Historia de la Ciencia y de la Técnica de Aragón. Facultad de Ciencias (Matemáticas), 1995), 178-179.

${ }^{7}$ En las citas se ha respetado la ortografía original.

8 José Odriozola, Curso Completo de Matemáticas Puras. Tomo I: Aritmética y Álgebra Elemental (Madrid, Imprenta que fue de García, 1827). Prólogo.

9 Odriozola, Curso Completo de Matemáticas Puras, tomo I, VI.
} 
Esta obra consta de cuatro tomos, en los cuales presentamos los tratados que en el dia forman el total de las Matemáticas puras por el orden mismo con que se han de estudiar [...]. Este orden sucesivo de materias hace que la obra pueda servir de testo en todos los establecimientos donde se enseñan Matemáticas, ya para los que solo necesitan ciertos conocimientos elementales de ellas, ya también para los que hayan de abanzar mas en la carrera de la ciencia. ${ }^{10}$

El libro analizado corresponde a la primera edición del Curso Completo De Matemáticas Puras. Tomo III: Álgebra Sublime y Geometría Analítica, impresa en Madrid en la «Imprenta que fue de García», en 1829, aunque los tomos I y II datan de 1827.

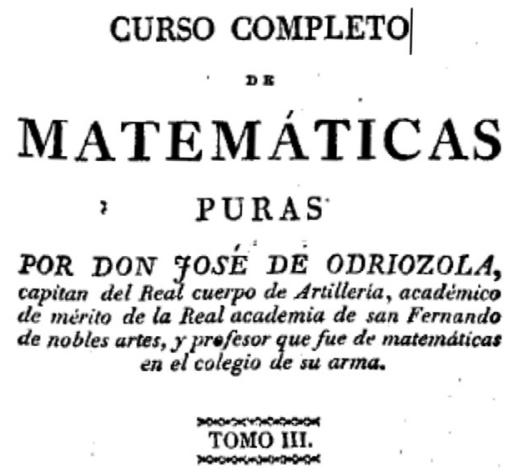

\section{ÁLGEBRA SUBLIME \\ Y GEOMETRÍA ANALIÍTICA.}

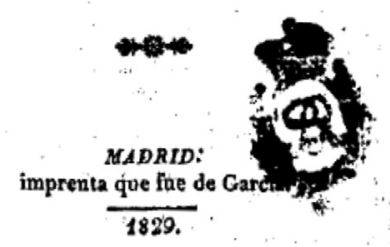

Biblioteca Nacional de España 园

Ilustración 1. Carátula del libro.

10 Odriozola, Curso Completo de Matemáticas Puras, tomo I, VI. 
El libro consta de 337 páginas de las cuales dedica 158 a la Geometría Analítica que está recogida en el «Tratado V» titulado «Geometría analítica o Aplicación del Álgebra a la Geometría». Este segundo término aparece en la mayoría de las obras de Geometría Analítica de este siglo, en ocasiones de forma indistinta al término Geometría Analítica, como es el caso, y en otros distinguiendo entre dos formas distintas de hacer Geometría Analítica: una más próxima a la geometría de Descartes, que hemos descrito brevemente al principio, y otra en la que se hace uso de los sistemas de coordenadas, muy similar a la actual. ${ }^{11}$ Odriozola distingue entre estas dos formas de hacer geometría hablando de «Ecuaciones determinadas de la Geometría» (cap. 1) en el primer caso y «Ecuaciones indeterminadas de la Geometría» (cap. 2) en el segundo. Todo ello englobado dentro de la geometría plana, dedicando otro apartado a la geometría del espacio.

En este artículo trataremos algunos aspectos estudiados en esos dos primeros capítulos.

\section{LA GEOMETRÍA ANALÍTICA DE ODRIOZOLA}

Como hemos comentado, en este texto encontramos dos maneras muy diferentes de hacer Geometría Analítica, que el autor separa en los capítulos 1 y 2 citados en el punto anterior. En la primera, Odriozola muestra una forma de resolver problemas geométricos en la que el Álgebra se aplica a la Geometría de manera similar a como se hace con la Aritmética, con la diferencia de que los números y las letras de las ecuaciones representan segmentos y no cantidades. Esto conlleva, como comentamos en la introducción, dos limitaciones importantes: en primer lugar, que las ecuaciones deben ser homogéneas y, en segundo lugar, el problema de la interpretación de las soluciones negativas. Por otra parte, una vez obtenida la solución algebraica, ésta se construye con regla y compás. Todos estos elementos se encuentran desarrollados en la obra.

La segunda manera de hacer Geometría Analítica, que aparece bajo el epígrafe «Ecuaciones indeterminadas de la Geometría» se basa en el

\footnotetext{
${ }_{11}$ Isabel M. Sánchez, «La Geometría Analítica en los libros de texto para secundaria y universidad en España en el siglo XIX» (Tesis doctoral, Universidad de Salamanca, 2015).
} 
concepto de lugar geométrico y se utilizan sistemas de coordenadas para determinar un punto en un plano.

\section{Ecuaciones determinadas de la Geometría}

Odriozola comienza este tema con una lección preliminar en la que explica la importancia de la aplicación del Álgebra a los problemas geométricos, pero sin menospreciar las demostraciones propias de la Geometría Elemental, y los pasos necesarios para llevar a cabo esta ayuda mutua.

Cuando aborda el papel que ocupa el Álgebra y los asuntos que trata advierte acerca de una de las limitaciones que ya se han comentado:

Toda espresion de Álgebra ó de Aritmética manifiesta conceptos acerca de cantidades, sea cualquiera la especie á que se concreten; de suerte que cada ecuacion de las que se ha dado a conocer en el cálculo general, se puede referir á la especie que convenga al calculador, salvo siempre la homogeneidad, y por consiguiente á las particulares de la estension. ${ }^{12}$

Señala la importancia de aplicar el Álgebra a la Geometría resaltando el papel de Descartes en esta rama:

Por mucho tiempo se abstuvieron los geómetras de emplear el Álgebra en las cuestiones indeterminadas de Geometría, sin embargo de ser un ramo estenso y más fecundo en frutos, que el de las determinadas; hasta que Descartes introdujo esta novedad que en los últimos tiempos ha ocupado talentos sublimes. A los modernos debemos innumerables verdades recientemente descubiertas; con que se han enriquecido los tesoros de la Geometría, creando nuevas ciencias, y metodizando las fisicas, de manera que hay en nuestros dias cuerpos muy estensos de doctrinas evidentes que por la análisis indeterminada se han formado en dichos ramos. ${ }^{13}$

\footnotetext{
12 José Odriozola, Curso Completo de Matemáticas Puras. Tomo III: Álgebra Sublime y Geometría Analítica (Madrid, Imprenta que fue de García, 1829), 169.

${ }_{13}$ Odriozola, Curso Completo de Matemáticas Puras, tomo III, 179.
} 
Pero no menosprecia las demostraciones propias de la Geometría elemental, pues:

en ellas la evidencia se hace sensible con objetos; y asi el método antiguo de los creadores se conserva para esplicar las verdades fundamentales, en que se apoya el cálculo para deducir otras nuevas, pero interponiendo desde los principios el auxilio del Algebra cuando fuere oportuno á beneficio de la claridad. ${ }^{14}$

Tras esto, pasa a explicar las aplicaciones del Álgebra a la Geometría y viceversa:

2. Desde ahora vamos á tratar de las reciprocas aplicaciones de una ciencia á la otra; y por el resultado se formará el juicio de que mas necesita del Algebra la Geometría, que de ésta la primera.

Nuestro actual asunto se divide en dos como la Geometría elemental; el primero es Algebra en la Geometría plana; y el segundo, Álgebra en la Geometría del espacio, desempeñando en ambas partes lo que exigen las aplicaciones recíprocas que hemos indicado, y estan resumidas en dos proposiciones generales: $1{ }^{a}$ cifrar en lenguaje de cálculo las cuestiones y resolverlas: $2 .^{a}$ construir espresiones del cálculo, ó traducirlas á lenguaje de figuras geométricas. ${ }^{15}$

Vemos aquí la manera de trabajar que apuntábamos en la introducción: se identifican las cantidades presentes en el problema, que en este caso serán las longitudes de los segmentos, se traducen a expresiones algebraicas y una vez resuelta la ecuación correspondiente se construyen geométricamente las soluciones. Da también unas orientaciones generales de cómo hacer las construcciones:

Para las construcciones se traza la escala (Geom. elem. 38), en ella se toman las unidades que contienen las cantidades conocidas, y la figura descrita oportunamente con éstas ha de manifestar las incógnitas, como se hizo ya en la Geometría elemental cuando se hallaron cuartas y medias proporcionales á rectas dadas. ${ }^{16}$

\footnotetext{
${ }^{14}$ Odriozola, Curso Completo de Matemáticas Puras, tomo III, 180.

15 Odriozola, Curso Completo de Matemáticas Puras, tomo III, 180.

16 Odriozola, Curso Completo de Matemáticas Puras, tomo III, 180.
} 
Añade que también en la Trigonometría se encontraron ejemplos «del modo con que se cifran problemas de la estension en lenguaje del Álgebra», y termina recordando la regla general para pasar del lenguaje geométrico al algebraico «que consiste en suponerlas resueltas (las cuestiones), y nombrando con cifras propias las cantidades, escribir la oracion para despues resolver segun los principios del cálculo». ${ }^{17}$

Incluimos uno de los problemas resueltos por el autor para mostrar de forma más concreta los pasos que sigue para resolverlo y cómo aplica el álgebra a la geometría: «V. ${ }^{\circ}$ Dado un polígono $\mathrm{ABCDF}$..., construir otro semejante cuya área esté con la primera en la razon de números $\frac{n}{m} »$.

Odriozola, a diferencia de otros autores de la época, comienza resolviendo el problema mediante métodos de la Geometría elemental, para hacerlo después con el álgebra.

Establece las condiciones que tienen que cumplir los lados homólogos de ambos polígonos: «Hay que hallar un lado $A B^{\prime}$ homólogo de $A B$ : y para ello, las dos condiciones de ser las áreas como los cuadrados de lados homólogos por su naturaleza, y como las líneas ó números $n, m$ por el dato, producen la relación $\frac{A B^{\prime 2}}{A B^{2}}=\frac{n}{m} \|^{18}$

Para construir la solución, recuerda una propiedad de los lados de un triángulo rectángulo basada en el teorema del cateto:

Debiendo primero resolver el problema gráficamente; recuérdese que la perpendicular $A J$ bajada desde el vértice del ángulo recto á la hipotenusa $\mathrm{BB}^{\prime}$, divide á ésta en dos partes $\mathrm{BJ}$, JB' cuya razón es (Geom. elem. 83) $\frac{A B^{\prime 2}}{A B^{2}}=\frac{J B^{\prime}}{J B}$

Por otra parte, prolongados los catetos y la perpendicular, los segmentos de todas las paralelas á BB' están en la razón $\frac{J B^{\prime}}{J B} \cdot{ }^{19}$

\footnotetext{
17 Odriozola, Curso Completo de Matemáticas Puras, tomo III, 181.

18 Odriozola, Curso Completo de Matemáticas Puras, tomo III, 195.

19 Odriozola, Curso Completo de Matemáticas Puras, tomo III, 196.
} 


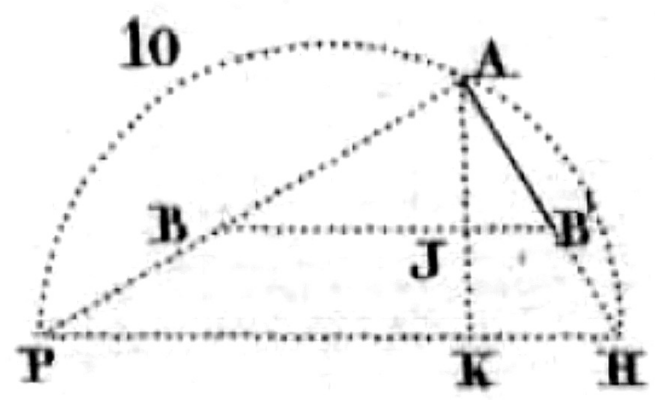

Ilustración 2. Figura 10. Odriozola, 1829.

Esta propiedad se utiliza para construir el lado buscado $A B^{\prime}$, pero lo hace fuera del polígono y luego lo traslada a él:

Tírese pues una recta indefinida y tomando en ella las partes $\mathrm{PK}=\mathrm{m}, \mathrm{KH}=\mathrm{n}$, constrúyase sobre el diámetro $\mathrm{PH}$ el semicírculo, y levántese en $\mathrm{K}$ perpendicular. Fórmese el triángulo rectángulo $\mathrm{PAH}$; y después de trasladar al cateto $\mathrm{AP}$ el lado $\mathrm{AB}$ del polígono dado, diríjase $\mathrm{BB}^{\prime}$ paralela á $\mathrm{PH}$, y será $\mathrm{AB}$ ' el lado que se busca homólogo de $\mathrm{AB} .{ }^{20}$

Esto lo hace con cada lado del polígono hasta que se obtiene el que se pretende:

Para construir el nuevo polígono conforme á lo espuesto (Geom. elem. 105), se hallan los demás lados por cuartas proporcionales; ó bien sobre el mismo polígono empezando desde $B^{\prime}$, se trazan $B^{\prime} C, C^{\prime} D^{\prime}[\ldots]$ paralelas á los respectivos lados de $A B C[\ldots]$ que serán cortadas debidamente por las diagonales $A C$, $A D,[\ldots] .{ }^{21}$

20 Odriozola, Curso Completo de Matemáticas Puras, tomo III, 196.

${ }^{21}$ Odriozola, Curso Completo de Matemáticas Puras, tomo III, 196. 


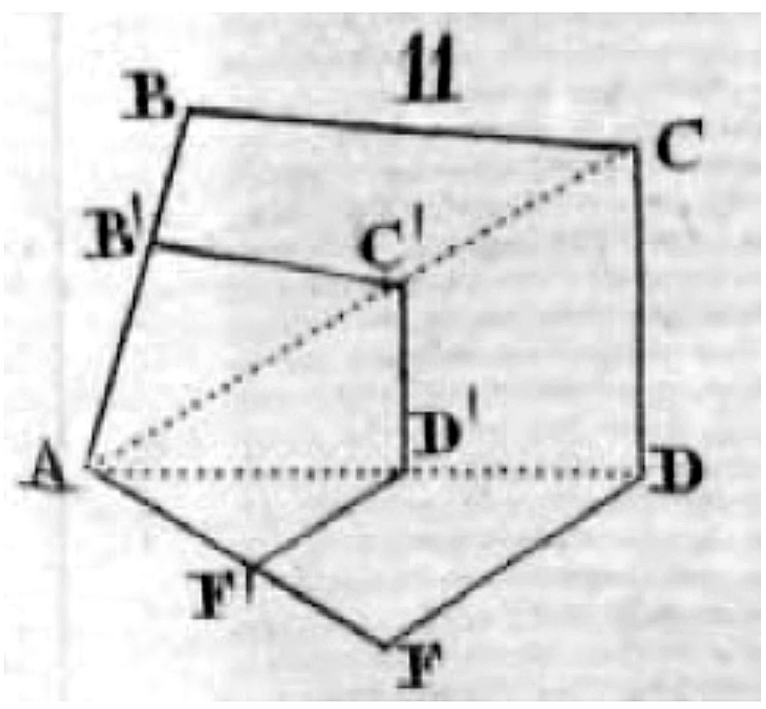

Ilustración 3. Figura 11. Odriozola, 1829.

Después de explicar la construcción desde el punto de vista de la Geometría, obtiene la solución utilizando el Álgebra:

La análisis manifiesta el resultado del mismo problema con mucha presteza; pues con las notas $\mathrm{AB}=\mathrm{q}$ y de su homólogo desconocido $A B^{\prime}=x$, el problema es $\frac{x^{2}}{q^{2}}=\frac{n}{m}$, y la solución $x=\frac{q}{m} \sqrt{m n}$.

Se construye elevando primeramente la perpendicular $\mathrm{KA}=\sqrt{\mathrm{mn}}$ en el circulo cuyo diámetro es $\mathrm{PK}+\mathrm{KH}=\mathrm{m}+\mathrm{n}$, para después hallar una cuarta proporcional $\mathrm{x}$ á las rectas $\mathrm{q}, \sqrt{\mathrm{mn}}, \mathrm{m} .{ }^{22}$

Veamos cómo explica Odriozola la «construcción de las ecuaciones determinadas» a lo que está dedicada la lección primera del capítulo I: «El asunto de esta leccion es traducir á lenguaje de figuras las mismas espresiones algébricas, que en la Geometría elemental se hallaron al traducir á lenguaje del cálculo las relaciones que tienen entre sí las líneas, las superficies y los volúmenes».23

22 Odriozola, Curso Completo de Matemáticas Puras, tomo III, 196.

${ }^{23}$ Odriozola, Curso Completo de Matemáticas Puras, tomo III, 182. 
Como otros autores de esta época, ${ }^{24}$ Odriozola insiste en la homogeneidad de las ecuaciones, utilizando de forma explícita una unidad para conseguirla, así como en la explicación de la construcción de las soluciones negativas de una ecuación. Sin embargo, en ningún caso dedica una sección aparte para explicar estos dos aspectos, sino que aparecen entremezclados en el texto con otras cuestiones, tanto en la teoría como en los problemas, por lo que analizaremos estos puntos de forma conjunta a la construcción de las fórmulas.

Odriozola hace un resumen de lo visto en Geometría elemental, incidiendo en la «dimensión» de las expresiones algebraicas:

1.Las líneas están espresadas en términos de una sola dimensión, tales como $x=a \pm b, x=n b$, siendo n número abstracto $x=\frac{a b}{c}, x=\sqrt{\left(a^{2} \pm b^{2}\right)}, x=\sqrt{(a b)}$ :

$2 .^{\circ}$ Las superficies están espresadas en productos de dos medidas, como $x=a b, x=a^{2}$, siendo $\mathrm{x}$ superficie, $\mathrm{y}$ los productos espresiones abreviadas de $\frac{x}{x^{\prime}}=\frac{a b}{a^{\prime} b^{\prime}}, \frac{x}{x^{\prime}}=\frac{a^{2}}{a^{2}}$ con las unidades $x^{\prime}, a^{\prime}, b^{\prime}$, de medida respectivas, de $x, a, b^{x_{\prime}}$.

3. ${ }^{\circ}$ Las espresiones de tres medidas dicen valores de los volúmenes, como $x=a b c, x=a^{3}$, abreviadas de $\frac{x}{x \prime}=\frac{a b c}{a b^{\prime} c^{\prime}}, \frac{x}{x^{\prime}}=\frac{a^{3}}{a^{\prime}{ }^{3}}$. $[\ldots] .25$

La homogeneidad de las expresiones es una cuestión clave para poder obtener expresiones con significado geométrico, y para conseguirlo señala:

De modo que siendo precisa la homogeneidad en las comparaciones, y por ello solamente comparables líneas entre si, superficies igualmente, así como los volúmenes; toda espresion de cantidades geométricas habrá de tener en los términos de ambos miembros igual número de factores lineales, fuera de los coeficientes abstractos, porque así vienen las espresiones halladas librándolas de forma fraccionaria. ${ }^{26}$

\footnotetext{
${ }^{24}$ Sánchez, «La Geometría Analítica en los libros de texto para secundaria y universidad en España en el siglo XIX».

25 Odriozola, Curso Completo de Matemáticas Puras, tomo III, 183.

26 Odriozola, Curso Completo de Matemáticas Puras, tomo III, 182.
} 
En el caso de la Trigonometría, para simplificar cálculos aparecen expresiones que no son homogéneas y aparentemente contradicen lo dicho, pero incorporando el radio, que se toma como unidad de medida, se observa que en realidad son expresiones homogéneas:

Por otra parte, en la Trigonometría, á fin de simplificar el cálculo, se suprime con frecuencia el radio por suponerle unidad de medida; y de aquí procede haber entonces en las ecuaciones mezclados términos de una dimensión con otros de dos y aun de tres; pero haciendo mención del radio $r$, se veria que entra en ellos con la potencia necesaria, para que todos fuesen de igual número de dimensiones. ${ }^{27}$

De forma análoga, en Geometría se hace uso de la unidad para obtener expresiones homogéneas, de manera que dichas expresiones puedan ser traducidas a formas geométricas:

Es pues necesaria cualidad de las notas algébricas para ser traducidas en figura de Geometría, el que sus términos de ambos miembros se completen hasta tener igual número de dimensiones cada uno, introduciendo en aquellos á quienes falten, la unidad lineal elevada á la potencia necesaria, para que la suma de esponentes de cada término sea el mismo: y una espresion así completada se llama homogénea. ${ }^{28}$

Una de las cuestiones que aparece en este libro, así como en otros de la época, es que se dota de significado geométrico a las expresiones algebraicas que surgen en la resolución de los problemas geométricos. Para ello se utilizan las operaciones entre segmentos y se construyen geométricamente estableciendo la relación geometría-álgebra. Estas construcciones las hace primero en el caso de que expresen longitudes, luego para áreas y por último para volúmenes. A continuación, se explicarán en detalle y se pondrán ejemplos de los tres casos.

En el primer caso Odriozola explica cómo sumar o restar segmentos y construir algunos tipos de cocientes y radicales:

\footnotetext{
27 Odriozola, Curso Completo de Matemáticas Puras, tomo III, 183.

28 Odriozola, Curso Completo de Matemáticas Puras, tomo III, 183.
} 
4. Para construir $x=a \pm b$, se tira una recta arbitraria y en ella se adopta, cuando no fuese dado, un punto $\mathrm{O}$ por origen de líneas: tomando después en la escala de partes iguales, que debe construirse de antemano, el valor de $a$, se traslada á la recta indefinida fijando en $\mathrm{O}$ la punta del compás: supuesto $a=O F$, se traslada también á continuación $F L=b$ si es $b$ positiva, y resulta $O L=a+b$. Mas cuando fuere $b$ negativa, se descontará $F L^{\prime}=b$ desde $\mathrm{F}$ retrocediendo ácia el origen, y quedará $O L^{\prime}=a-b .{ }^{29}$

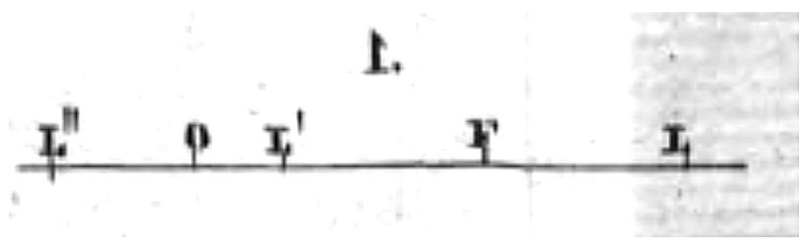

Ilustración 4. Figura 1. Odriozola, 1829.

También explica el caso en que $b>a$, y por tanto $a-b<0$ y de esta forma indica cómo tratar las cantidades negativas:

Puede suceder que sea $b>a$, de modo que, al retroceder desde $F$ ácia el origen, resulte $b=F L^{\prime \prime}$; en este caso x habrá pasado á ser menor que cero en la cantidad $O L^{\prime \prime}$, y será $O L^{\prime \prime}=-x=a-b$. Por esto, siempre que se haya de construir una línea espresada con signo negativo, es necesario trazarla desde el origen ácia la parte opuesta de la que ocuparía si fuese positivo el signo. ${ }^{30}$

La construcción de distintos cocientes se reduce a la de cuartas proporcionales a tres segmentos dados. El caso más sencillo es $x= \pm \frac{a b}{e}$.

5. $x= \pm \frac{a b}{e}$ es teorema de líneas proporcionales con la incógnita $x$. Trazadas las rectas $O M, O N$ formando cualquiera ángulo, tómense en $O M$ las partes $O D=e, O D^{\prime}=b$, y en $O N$ la parte $O B=a$. Diríjanse $D B, D^{\prime} B^{\prime}$ paralelas, y resultará $O B^{\prime}=x$ (Geom. elem. 38, III). ${ }^{31}$

\footnotetext{
29 Odriozola, Curso Completo de Matemáticas Puras, tomo III, 184.

30 Odriozola, Curso Completo de Matemáticas Puras, tomo III, 184.

31 Odriozola, Curso Completo de Matemáticas Puras, tomo III, 184.
} 


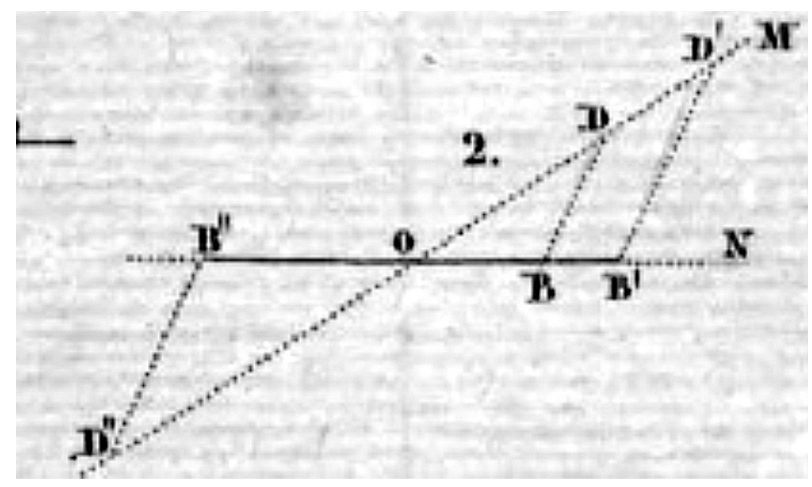

Ilustración 5. Figura 2. Odriozola, 1829.

En el caso en que una de las cantidades sea negativa también se explica el procedimiento de construcción de forma análoga.

[...] Si fuese negativa una de las cantidades $a, b, e$, lo será también $x$; y tomando las dos positivas ácia una parte del origen, y la negativa ácia el otro, en las líneas correspondientes, resultará á cada lado un triángulo, ambos semejantes, y el lado de uno de ellos será $-x$, como $O B^{\prime \prime}$ cuando $O D^{\prime \prime}$ fuere igual á la negativa de las dadas. ${ }^{32}$

En caso de cocientes más complejos los reduce al caso anterior, por ejemplo si $x=\frac{a b^{2} d}{e f g}$ se simplifica haciendo $\frac{a b}{e}=m, \frac{b d}{f}=n$, con lo que resulta $x=\frac{m n}{g}$, todos ellos cocientes que se construyen sucesivamente por el método explicado en el punto anterior.

También explica los casos en que hay polinomios en el cociente. Especialmente interesante es aquel en que el denominador es un polinomio, $x=\frac{a b}{e \pm f}$. En esta ocasión toma $k=e \pm f$, que construye como ha explicado antes, con lo que obtiene $x= \pm \frac{a b}{k}$ que también se sabe construir. Considera el caso de que el denominador sea negativo y el numerador positivo, con lo que $x$ también será negativo. Lo interesante de esta construcción es la reflexión que hace sobre cómo puede pasar un valor de positivo a negativo:

32 Odriozola, Curso Completo de Matemáticas Puras, tomo III, 184. 
Hasta aquí hemos considerado las líneas negativas, como si hubiesen llegado á tener este signo pasando por el valor cero desde positivas: así puede considerarse también $k$, pero no $x$; porque en el caso de $k=0$, sería $x=\frac{a b}{0}=\infty$ de modo que ha llegado $x$ de positiva á negativa pasando por el infinito. Conocemos ya dos modos diferentes de haber cambio de signo en la espresion de una línea, conformes á los que reconocimos en casos de ocurrir este accidente en la cantidad general (Algebra elemental 131). ${ }^{33}$

Esta reflexión también la hacen Mariano Zorraquín en su obra Geometría Analitico-Descriptiva (1819) ${ }^{34}$ y Alberto Lista en Elementos de Matemáticas Puras y Mistas (1825). ${ }^{35}$ Vemos que consideran las cantidades como continuas - como expresiones algebraicas que son, de hecho Odriozola nos remite al Álgebra- pudiendo tomar cualquier valor. La diferencia entre Zorraquín y Odriozola es que el primero utiliza esta propiedad como una característica de las cantidades que cambian de signo, para saber en un problema qué cantidades pueden pasar de positivas a negativas y dar nuevas interpretaciones de ese problema o de las soluciones negativas que aparecen. Odriozola, sin embargo, simplemente hace la reflexión cuando aparece el caso, pero no lo utiliza para resolver ningún problema.

También trata Odriozola la construcción de expresiones radicales:

10. Para construir la línea del valor que espresa $x= \pm \sqrt{a b}$ en que $x$ es media proporcional entre $a$ y $b$, tómese la recta $D F=a+b$, y descríbase un círculo cuyo diámetro sea $D F$ y elevando la recta $H G$ perpendicular en el punto $H$ de adición, la parte $H G$ ó $H G^{\prime}$ comprendida entre $H$ y la circunferencia, será la línea $x .^{36}$

\footnotetext{
33 Odriozola, Curso Completo de Matemáticas Puras, tomo III, 185.

${ }^{34}$ Isabel M ${ }^{\mathrm{a}}$ Sánchez Sierra y M $\mathrm{M}^{\mathrm{a}}$ Teresa González Astudillo, «La Geometría Analítico-Descriptiva de Mariano Zorraquín» HISTEMAT 2, no. 3 (2016): 200-228.

35 Isabel $\mathbf{M}^{\mathrm{a}}$ Sánchez Sierra y $\mathbf{M}^{\mathrm{a}}$ Teresa González Astudillo, «La geometría analítica de Alberto Lista». Épsilon - Revista de Educación Matemática 34, no. 95 (2017): 7-24.

36 Odriozola, Curso Completo de Matemáticas Puras, tomo III, 187.
} 


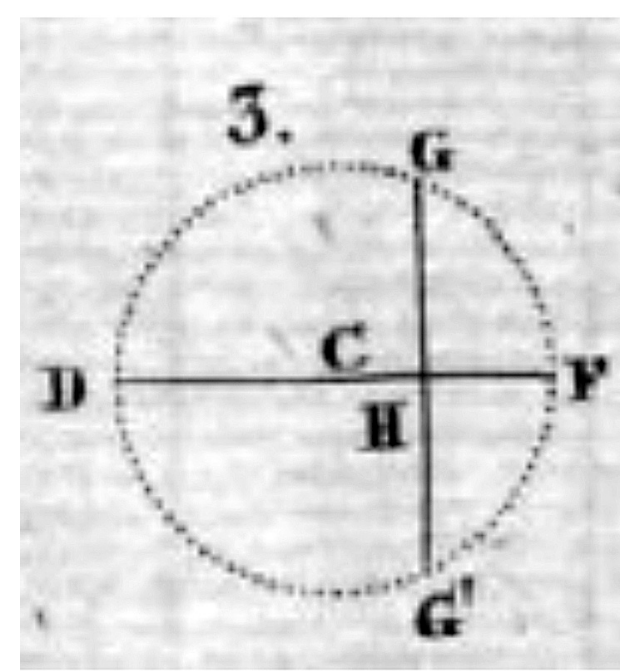

Ilustración 6. Figura 3. Odriozola, 1829.

Es decir, utiliza el teorema de la altura para construirlo.

11. Si se ofrece hallar el valor de la línea $x$ por la ecuación $x=\sqrt{\left(a^{2} \pm b^{2}\right)}$ ó $x^{2}=a^{2} \pm b^{2}$ sabemos que construyendo un triángulo rectángulo cuyos catetos sean $a$ y $b$, la hipotenusa será $x$ en caso de $b^{2}$ positivo; pero si este fuese negativo, $a$ será hipotenusa, $b$ un cateto y $x$ el otro. En el primer caso, construyendo con lados indefinidos el ángulo recto y fijando la punta del compás en el vértice $F$, se marcan los estremos de los catetos para trazar la hipotenusa $G H=x$. En el segundo caso, marcando el estremo $H$ del cateto $F H$ conocido desde $H$ con el radio $a$ se trazará un arco, $y$ éste cortará en $G$ el otro cateto $F G=x{ }^{37}$

37 Odriozola, Curso Completo de Matemáticas Puras, tomo III, 187. 


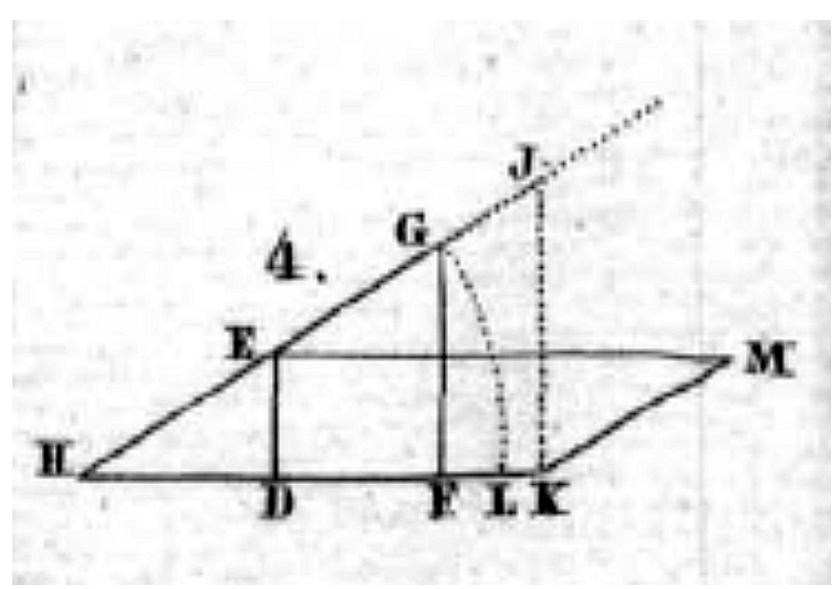

Ilustración 7. Figura 4. Odriozola, 1829.

Como en el caso de los cocientes pone ejemplos de radicales más complejos que se construyen reduciéndolos a los casos anteriores. Por ejemplo, en el caso $x=\sqrt{a^{2}+b^{2}+c^{2}+\ldots}$ hace $v^{2}=a^{2}+b^{2}$, construye $v, \mathrm{y}$ después $x=\sqrt{v^{2}+c^{2}} \cdot 38$

Además, analiza la ecuación $x^{2} \pm p x=q$, cuyas soluciones contienen expresiones radicales. Vemos aquí un ejemplo de uso explícito de la unidad para convertir la ecuación en homogénea. Después halla sus soluciones, cuya construcción se reduce a los casos anteriores:

4..$^{\circ}$ La espresion $x^{2} \pm p x=q$ exige completarse multiplicando el segundo miembro por $r=1$, con lo que viene á ser $x^{2} \pm p x=r q \mathrm{y}$ haciendo $r q=m^{2}$ la incógnita despejada es $x= \pm \frac{1}{2} \pm \sqrt{\left(m^{2}+\frac{1}{4} p^{2}\right)}$ fácil de construir por adición de las líneas $\pm \frac{1}{2}$ p y $\pm \sqrt{ }\left(m^{2}+\frac{1}{4} p^{2}\right) \cdot 39$

El uso de los teoremas de Geometría le sirve para realizar otras construcciones de las soluciones de la ecuación anterior sin necesidad de resolverla:

13. Los teoremas de Geometría elemental [...] también son aplicables á la construcción de raices en las ecuaciones de segundo grado, sin que sea necesario despejar la incógnita; pues, $x^{2} \pm p x=$

\footnotetext{
38 Odriozola, Curso Completo de Matemáticas Puras, tomo III, 187.

39 Odriozola, Curso Completo de Matemáticas Puras, tomo III, 187.
} 
$m^{2}$ equivale á $x(x \pm p)= \pm m^{2}$ la cual en todos los casos que envuelve se puede construir por las líneas proporcionales del círculo.40

Obsérvese la visión geométrica que tiene, que le permite ver en una ecuación una propiedad de la circunferencia. Por ejemplo, la expresión $x(x-p)=m^{2}$ se puede traducir a la propiedad de la circunferencia que dice que si una cuerda (en este caso $m$ ) y un diámetro $(x)$, tienen un punto común sobre la circunferencia, la cuerda es media proporcional entre el diámetro y su proyección sobre este $(x-p)$.

Además de las expresiones anteriores construye $x=\sqrt{m}$, que convierte en homogénea utilizando la unidad, $r=1$, con lo que se transforma en $x=\sqrt{r m}$, «que se construye como se ha dicho anteriormente».41 Pero además da formas particulares para construir $\sqrt{2}, \sqrt{3}, \sqrt{5}$ y $\sqrt{6}$.

Si es $m=2$ descríbase un círculo con el radio $r=1$ y el lado del cuadrado inscrito vale $\sqrt{2}$ (G. el. 101, II. ${ }^{\circ}$ ).

Si es $m=3$; el lado del triángulo equilátero inscrito en el mismo círculo del radio $r=1$ vale $\sqrt{3}$ como se sabe $\left(\mathrm{Geom} .101, \mathrm{I}^{\circ}\right)$.

Cuando sea $m=5$ con los catetos $A B=2, B C=1$ se construye el triángulo rectángulo $A B C$, en que es $A C=\sqrt{4+1}=\sqrt{5} .{ }^{42}$

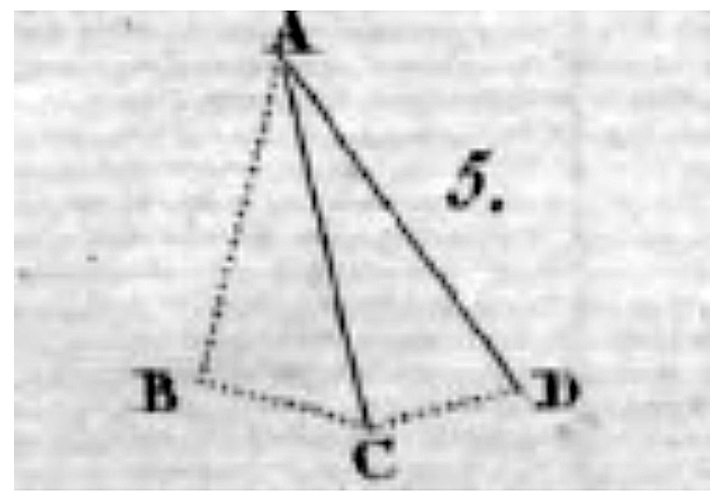

Ilustración 8. Figura 5. Odriozola, 1829.

\footnotetext{
40 Odriozola, Curso Completo de Matemáticas Puras, tomo III, 188.

41 Odriozola, Curso Completo de Matemáticas Puras, tomo III, 188.

42 Odriozola, Curso Completo de Matemáticas Puras, tomo III, 188.
} 
De forma análoga construye $\sqrt{6}$ utilizando como catetos $A C=\sqrt{5} \mathrm{y}$ $B C=1$, y añade que "por el mismo orden se pueden construir sucesivamente las raíces cuadradas de todos los números enteros».43

Para las ecuaciones de grado superior al segundo indica:

15. Dada una ecuación de grado superior al segundo, comprendida en la general $x^{m}+p x^{m-1}+q x^{m-2}+\& c$. y hallados analíticamente sus factores simples y los binarios, la construcción de sus raices de primero y segundo grado se reduce á practicar lo prevenido en los casos anteriores. ${ }^{44}$

En cuanto a la construcción de las «expresiones circulares» señala:

16. En las espresiones circulares $x=\frac{\operatorname{sen} a}{b}, v=\frac{\cos a}{d}$ hay que suplir el radio $r=1$; y de este modo pasan á ser $x=\frac{r \cdot \operatorname{sen} a}{b}, v=\frac{r \cdot \cos a}{d}$ en que $x$ y $v$ son cuartas proporcionales á tres rectas dadas. Para construirlas se traza con un radio $H G$ arbitrario el arco (ilustración 8); se toma el valor $G L$ del ángulo $a$ cuya línea trigonométrica es conocida, para tener $G F=\operatorname{sen} a, H F=\cos a$.

Sabiendo ya los valores de tres dimensiones de cada ecuacion, fácil es construir $x y v$ por el método (5) de cuartas proporcionales. 45

Vemos aquí, como ya habíamos dicho, el uso explícito de la unidad, que en otros autores de la época no se da. La citan y explican cómo debe usarse, pero generalmente la utilizan de forma implícita. ${ }^{46}$

Odriozola también explica la construcción de un área y un volumen:

17. [...] Para traducir á lenguage de Geometría la espresion $x=a b$, desde luego se advierte que $x$ representa una área, por constar de dos dimensiones el segundo miembro: y sabemos por los elementos que tanto el rectángulo construido con los lados $a$ y $b$,

\footnotetext{
43 Odriozola, Curso Completo de Matemáticas Puras, tomo III, 188.

${ }^{44}$ Odriozola, Curso Completo de Matemáticas Puras, tomo III, 188.

45 Odriozola, Curso Completo de Matemáticas Puras, tomo III, 189.

46 Sánchez, «La Geometría Analítica en los libros de texto para secundaria y universidad en España en el siglo XIX».
} 
como el paralelogramo de ángulos arbitrarios que tenga por base una de estas dimensiones y por altura la otra, como también el triángulo que tenga por base la una y por altura el duplo de la otra, constan de un mismo número de unidades cuadradas espresado en el producto $a b$. De suerte que, construyendo así cualquiera de dichas figuras, queda resuelto el problema. ${ }^{47}$

Propone una expresión trigonométrica que aparentemente es de dimensión tres (es decir, un volumen), pues tiene tres factores, pero que resulta ser de dos (o sea, un área) al hacer uso de la unidad, que en este caso es el radio.

Si la espresion que se ofrece construir fuese $x=a \cdot b \cdot \operatorname{sen} u$ á primera vista se nota que pertenece á las de dos dimensiones, porque falta en el denominador el radio $r=1$ á quien se refieren todas las líneas trigonométricas. Completándola y haciendo $p=\frac{a \operatorname{senu}}{r}$ hay que construir finalmente $x=p b .{ }^{48}$

Y explica su construcción: «Supuestos $H G=r, G F=\operatorname{senu}, H E=a, H K=b$,

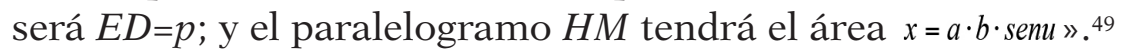

Después estudia dos casos particulares de áreas: la primera, el caso en que $\operatorname{sen} u=1$, en que la expresión se convierte en $x=a b$, "área del rectángulo cuyos lados son $a, b$ ». La segunda, la expresión de grado dos $x=\frac{a^{2} b c+b d f g-d^{3} h}{c d+e h}$, que simplifica convirtiéndola, tras diversas transformaciones, en producto de dos expresiones de grado uno, que llama $g$ y $w$, respectivamente, «luego, $x$ es un rectángulo que tiene por base y altura las líneas $g, w » .^{50}$

Por último, se ocupa de la construcción de volúmenes. Uno de los casos que propone es el relativo a la expresión que transforma, de manera similar al anterior, en $x=r^{2} w$, que «expresa el volumen de un paralepípedo, que tenga por base el cuadrado $r^{2}$ y por altura la línea $w »$ y del

\footnotetext{
47 Odriozola, Curso Completo de Matemáticas Puras, tomo III, 189.

48 Odriozola, Curso Completo de Matemáticas Puras, tomo III, 189.

49 Odriozola, Curso Completo de Matemáticas Puras, tomo III, 190.

50 Odriozola, Curso Completo de Matemáticas Puras, tomo III, 190.
} 
que dice que «la construcción es fácil por las reglas de la Geometría en el espacio», ${ }^{51}$ y explica cómo construir la expresión $x=a b c$ :

Si se hubiera de construir la figura cuyo volumen espresa $x$ en $x=a b c$, es arbitraria la combinación de dimensiones para la base, y la figura paralelógrama de ésta, é igualmente la inclinación del paralelepípedo respecto de la base. Es preferible sin embargo la figura rectangular por ser mas determinada, y también porque $x=a b c$ se puede considerar como caso particular de $x=a b c \cdot \operatorname{senu}$, cuando el seno es igual al radio, y aquí sen $u=1 . .^{52}$

Y para el caso general:

Si la ecuación que se ha de construir fuere la mas general $x=a b c$. senu se halla primeramente la línea $p=a \cdot \operatorname{senu}$; y resulta el paralelogramo $p b$ ó $p c$ para la base, que será cualquiera de las caras del paralelepípedo, quedando para la altura de éste la otra dimensión de $p b c$.

Incluimos a continuación dos de los problemas resueltos por el autor en los que se aplican algunas de las construcciones explicadas anteriormente. Se puede observar también cómo trata las soluciones negativas de los problemas, así como la necesidad de utilizar expresiones homogéneas o, al menos, aquellas que representen una entidad geométrica:

III. ${ }^{\circ}$ Cortar la recta $\mathrm{AB}$ en media y estrema razón, es decir, en dos partes de manera que la mayor sea media proporcional entre la menor y el todo.

En este problema encontramos la construcción de un radical y de una solución negativa.

Como en el resto de problemas, hace mención a la solución geométrica, pero en este caso no la incluye, pues señala que ya se halló en la parte de Geometría. Se resuelve el problema por métodos algebraicos, que incluimos a continuación:

\footnotetext{
51 Odriozola, Curso Completo de Matemáticas Puras, tomo III, 191.

52 Odriozola, Curso Completo de Matemáticas Puras, tomo III, 191.
} 
Para resolver analíticamente la cuestión, sea $A B=a, A D=x$, de que resulta $D B=a-x$, y el problema cifrado en $\frac{a}{x}=\frac{x}{a-x}$.

Prepárese por las reglas del Algebra esta ecuación para resolverla, y despejando $x$ se hallará $x=-\frac{1}{2} a \pm \sqrt{\left(a^{2}+\frac{1}{4} a^{2}\right)} \cdot{ }^{53}$

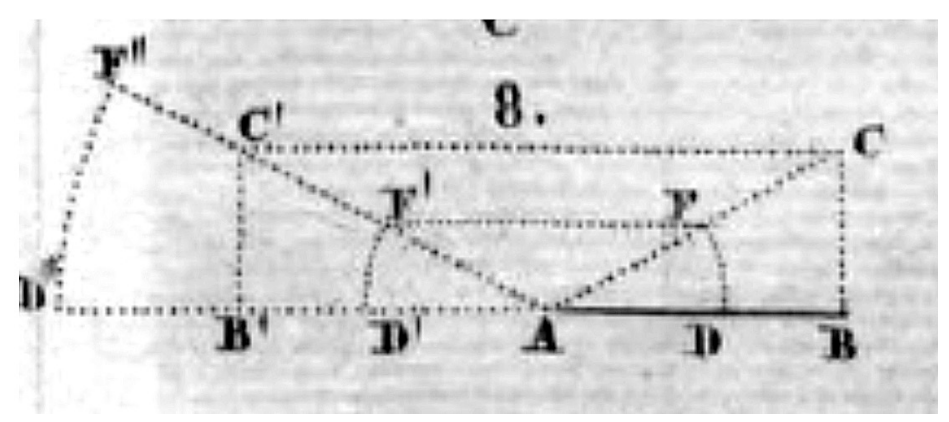

Ilustración 9. Figura 8. Odriozola, 1829.

A continuación, construye la solución geométricamente:

Habiéndola de construir, elévese la perpendicular $B C=\frac{1}{2} a \quad \mathrm{y}$ será $A C=\sqrt{\left(a^{2}+\frac{1}{4} a^{2}\right)}$. Tomando en la recta $A C$ la parte $C F=C B$, y en la recta $A B$ la parte $A D=A F$, se tiene $A D=-\frac{1}{2}+\sqrt{\left(a^{2}+\frac{1}{4} a^{2}\right)} \cdot{ }^{54}$

Y construye la negativa, aunque en este caso la desecha:

Para construir la otra raiz hállese $A C^{\prime}=-\sqrt{\left(a^{2}+\frac{1}{4} a^{2}\right)}$, y por análogas operaciones ácia esta parte, será $A F^{\prime \prime}=A D^{\prime \prime}$ del valor $x=-\frac{1}{2}-\sqrt{\left(a^{2}+\frac{1}{4} a^{2}\right)}$; sin embargo de que esta raiz no hace al caso, pues el problema exige que el punto de división se halle entre $A$ y $B .55$

53 Odriozola, Curso Completo de Matemáticas Puras, tomo III, 193.

54 Odriozola, Curso Completo de Matemáticas Puras, tomo III, 194.

55 Odriozola, Curso Completo de Matemáticas Puras, tomo III, 194. 
Haremos dos observaciones sobre la resolución de este problema, en relación con otros autores de la época. ${ }^{56}$ En primer lugar, es usual que una vez construida la solución algebraica se comprueba si, en efecto, es la correcta; en este caso que la construida es la cuarta proporcional buscada. Odriozola sabe que es la solución del problema porque lo ha resuelto antes geométricamente, pero no comprueba que, en efecto, coincide con la solución algebraica.

Por otra parte, la solución negativa no se suele desechar, como es el caso, sino que se interpreta utilizándola, generalmente para dar una versión más general del problema. ${ }^{57}$

IV. ${ }^{\circ}$ Dada la recta DF, dividirla en dos partes con las cuales ha de formarse rectángulo igual á un cuadrado conocido $\mathrm{m}^{2}$.

En este problema se lleva a cabo la construcción de un área. Obsérvese que toma como área $m^{2}$, y no una cantidad $m$, para conseguir así que la ecuación obtenida sea homogénea, sin necesidad de utilizar la unidad.

Según su costumbre, Odriozola, primero hace la construcción por medios puramente geométricos:

Después de construir el semicírculo sobre el diámetro $D F$, y elevar en $D$ la perpendicular definida, tómese en ella $D L=m$; la recta $L G$ paralela al diámetro corta la circunferencia en dos puntos $G, G^{\prime}$ y las perpendiculares $G H, G^{\prime} H^{\prime}$ cortarán al diámetro en los puntos $H, H^{\prime}$ según está pedido; y bastaba uno de ellos para la solución. ${ }^{58}$

\footnotetext{
56 Sánchez, «La Geometría Analítica en los libros de texto para secundaria y universidad en España en el siglo XIX».

${ }^{57}$ Isabel M. Sánchez Sierra y M. Teresa González Astudillo, «La geometría analítica en España durante el siglo XIX: estudio de las soluciones negativas de una ecuación", Enseñanza de las ciencias 35, no. 3 (2017): 89-106. https://doi.org/10.5565/rev/ensciencias. 2348.

58 Odriozola, Curso Completo de Matemáticas Puras, tomo III, 194.
} 


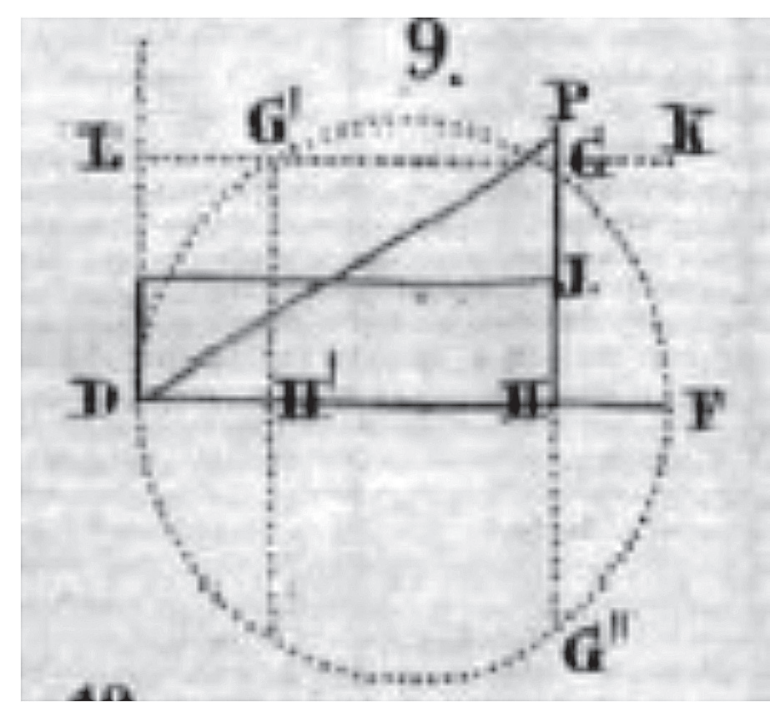

Ilustración 10. Figura 9. Odriozola, 1829.

Seguidamente lo resuelve con ayuda del Álgebra:

Tratándose de resolver el problema propuesto IV. ${ }^{\circ}$ analíticamente, sean $D F=a, D H=x$, y por ello $H F=a-x$; la cuestión cifrada en este lenguaje será $x(a-x)=m^{2}$ de donde $x=\frac{1}{2} \pm \sqrt{\left(a^{2}+\frac{1}{4} a^{2}\right) .{ }^{59}}$

En este caso no desarrolla la construcción geométrica de la solución, pero sí da su interpretación geométrica:

Constrúyase $x$ según manifiesta la espresion, y se tiene el lado del rectángulo en que será el otro $a-x$, con la misma área que un cuadrado $m^{2}$; ó una dimensión del paralelógramo de la misma área que tenga por base y altura las líneas $x, a-x$; y aun si se quiere, de un triángulo equivalente que tenga por base y altura las respectivas lineas $x y 2(a-x)$. Con facilidad se nota en la solución que el problema es imposible cuando los datos incurran en el caso $>\frac{1}{2} a \cdot{ }^{60}$

Tras esto plantea el problema inverso y lo resuelve (Ilustración 10: Figura 9):

59 Odriozola, Curso Completo de Matemáticas Puras, tomo III, 194.

${ }_{60}$ Odriozola, Curso Completo de Matemáticas Puras, tomo III, 195. 
Inversamente; si, dado un paralelógramo de las dimensiones $x$ y $a-x$, se quiere un cuadrado de igual área, está cifrada la cuestión en la misma fórmula pero entonces es $m$ la incógnita, $a$ la suma de las dos dimensiones del paralelógramo dado, y $x$ una de ellas por supuesto conocida.

La solución viene á ser $m= \pm \sqrt{x(a-x)}$ que se construye formando el círculo con el diámetro $a$, y elevando una perpendicular desde el punto en que se unen sus partes $x$ y $a-x$. En este caso $H G$, $H G^{\prime \prime}$ iguales de signos contrarios satisfacen, y el problema no es posible cuando $\mathrm{x}>\mathrm{a} \cdot{ }^{61}$

Obsérvese que la construcción geométrica de la solución algebraica tiene muchas similitudes a la construcción de la solución obtenida únicamente por métodos geométricos.

Tras esto obtiene la siguiente conclusión, que apoya el empleo del Álgebra en la resolución de los problemas geométricos:

En vista de la reciprocidad de los dos problemas cifrados en una misma ecuación, se recuerda lo observado en los principios del Algebra acerca de que una fórmula espresa diversos problemas, según se trate como incógnita una ú otra de las cantidades que entran en ella. ${ }^{62}$

\section{ECUACIONES INDETERMINADAS DE LA GEOMETRÍA}

Por otra parte, y como ya hemos señalado, Odriozola también estudia la aplicación del Álgebra a la Geometría mediante el uso de sistemas de coordenadas, dedicando el capítulo II al estudio de las ecuaciones de primer y segundo grado. En este artículo describiremos brevemente los sistemas de coordenadas que considera y pondremos como ejemplo el estudio de las ecuaciones de primer grado correspondientes a las rectas.

En cuanto a los sistemas de referencia, utiliza lo que él llama sistemas de coordenadas rectas, que pueden ser oblicuas y rectangulares, y las coordenadas polares; así como los cambios entre ellos.

\footnotetext{
${ }_{61}$ Odriozola, Curso Completo de Matemáticas Puras, tomo III, 195.

62 Odriozola, Curso Completo de Matemáticas Puras, tomo III, 195.
} 
Justifica la necesidad y el objeto de los sistemas de referencia que no ha utilizado en el capítulo anterior:

21. En los problemas de una sola incógnita el objeto es, como se ha visto, determinar un punto $K$ de una recta fija $O H$, y basta para ello saber dirigir otra recta $B K$ á quien pertenezca también el punto $K$; pues la intersección cumple sola con dichas condiciones. Pero si el punto $K$ estuviese aislado en el plano fuera de toda recta de posición conocida, es preciso entonces para situarle saber cómo se dirigirán dos rectas en que se halle simultáneamente, lo cual exige dos ecuaciones finales.

Con este motivo, y para facilitar medios de investigación, se establecen sistemas de líneas fijas á quienes puedan referirse todas las otras del plano, en cuanto á la posición que tienen respecto de aquellas. ${ }^{63}$

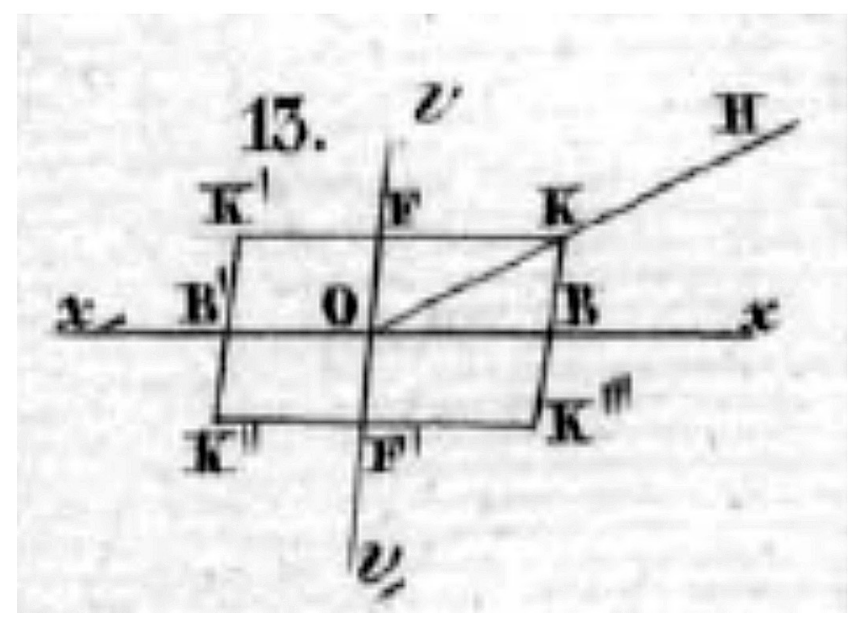

Ilustración 11. Figura 13. Odriozola, 1829.

Explica cómo, a partir de dos ejes de coordenadas rectas se debe dar la posición de un punto:

${ }_{63}$ Odriozola, Curso Completo de Matemáticas Puras, tomo III, 198. 
Dirigiendo desde un punto $O$, arbitrariamente elegido, las rectas $O x, O v$ de modo que formen cualquiera ángulo, y convenidos en que todo punto $K$ del plano ha de ser intersección de rectas $B K, F K$, respectivamente paralelas á $O v, O x$; sólo habrá que hallarlas distancias $O B=F K=x, O F=B K=v$, sin que sea necesario complicar las espresiones con datos acerca de la respectiva dirección de las lineas $F K, B K{ }^{64}$

Y continúa dando la definición de ejes coordenados y sus nombres, de forma muy similar a como se hace en la actualidad:

Las rectas $O x, O v$ fijas se llaman ejes coordenados, el uno de las $x$, el otro de las $v$; las partes $O B, O F$ se dicen abscisa y ordena$d a$, y ambas coordenadas del punto $K$. Comunmente se dice que $O x$ es el eje de abcisas, y $O v$ de ordenadas: el punto $O$ es origen de coordenadas. ${ }^{65}$

Obsérvese que, aunque los ejes reciben los mismos nombres que en la actualidad, la representación simbólica del de ordenadas es $O v$, y no Oy como es habitual hoy en día.

Tras esto explica el signo que toman las coordenadas de los puntos dependiendo de su posición respecto a los ejes, y concluye diciendo que «si viene doble solución para una de las coordenadas $x$, $v$, hay dos puntos determinados; y si viene doble solución para las dos, hay determinados cuatro puntos». ${ }^{66}$

Por otra parte:

Si resultasen las ecuaciones finales $\pm \mathrm{x}=\mathrm{OB}, \pm v=0$, el punto determinado es $B$ ó $B^{\prime}$ en el eje de abscisas. Si las ecuaciones fuesen $\pm \mathrm{x}=0, \pm \mathrm{v}=\mathrm{OF}$, el punto determinado es $F$, ó $F^{\prime}$ en el eje de ordenadas; éste es el caso de los problemas con una sola incógnita $v .67$

\footnotetext{
${ }_{64}$ Odriozola, Curso Completo de Matemáticas Puras, tomo III, 198.

65 Odriozola, Curso Completo de Matemáticas Puras, tomo III, 199.

66 Odriozola, Curso Completo de Matemáticas Puras, tomo III, 199.

${ }_{67}$ Odriozola, Curso Completo de Matemáticas Puras, tomo III, 199.
} 
Obsérvese que el signo lo lleva la variable, $x$ o $v$ y no el segmento. Incluso en la figura 13 vemos que en los ejes también aparecen $x,-x, v,-v$, aunque él pone el signo debajo de la letra y no a la izquierda, tal como explica en el punto 32, en el que recoge algunas convenciones de notación que utilizará.

También define las coordenadas polares:

El sistema polar consiste en medir los grados de un arco de círculo $B M$, contado desde un punto $B$ fijo de la circunferencia, y al mismo tiempo la longitud de la recta $O K$ llamada radio vector; de modo que siendo la circunferencia línea de las $u$, y línea de las $t$ la recta indefinida $O M$ que girando sobre su estremo $O$ llamado polo es capaz de todas inclinaciones respecto de la fija $O B$, el punto $K$ estará determinado cuando sean conocidos el arco $B M$ en grados y la longitud $O K$, por las ecuaciones $u=a, t=b .{ }^{68}$

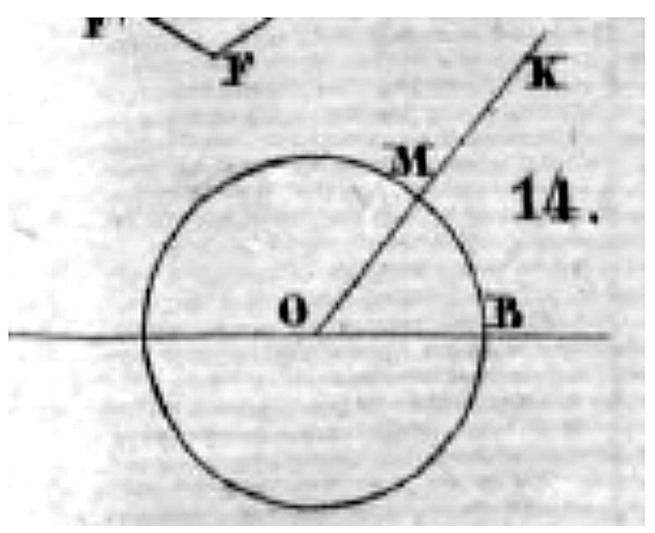

Ilustración 12. Figura 14. Odriozola, 1829.

Odriozola explica cómo pasar de unos sistemas de referencia a otros, indicando tres tipos de cambios entre sistemas de coordenadas rectangulares u oblicuas: «1. ${ }^{\mathrm{a}}$ Trasladar el oríjen $O$ á otro punto, quedando las nuevas coordenadas $O^{\prime} x^{\prime}, O^{\prime} v^{\prime}$ paralelas á las primitivas. 2. ${ }^{a}$ Sobre el oríjen $O^{\prime}$ variar el ángulo de las coordenadas primitivas $O^{\prime} x$ y $O^{\prime} v$. 3. ${ }^{a}$ Hacer á un tiempo las dos novedades, que es la mayor transformación posible».69 También considera el paso de coordenadas rectangulares a polares.

\footnotetext{
68 Odriozola, Curso Completo de Matemáticas Puras, tomo III, 199.

${ }_{69}$ Odriozola, Curso Completo de Matemáticas Puras, tomo III, 201.
} 
La ecuación de la recta se establece en la lección II titulada «Análisis geométrica de la ecuacion (sic) de primer grado con dos variables».

A partir de una ecuación de primer grado general determina la ecuación explícita de la recta, aunque él no las denomina así:

30. La ecuación de primer grado con las variables $x, v$ es $A v+B x+C=0 ; y$ despojando de coeficiente á su primer término, recibe la forma $v=\frac{B}{A} x+\frac{C}{A}$, en que son positivos ó negativos $A, B$, $C$. Si es $C=0$ queda la ecuación reducida á, $v=\frac{B}{A} x$. Suponiendo $\frac{B}{A}=a, \frac{C}{A}=b$ serán las ecuaciones de ambos casos $v=a x+b ;$ $v=a x+b ; 7^{70}$

Para la última de estas ecuaciones, $v=a x$, deduce que representa una recta y determina el significado de la constante $a$ :

Construida la recta $O M$, y bajadas desde sus puntos las $M P$, $M^{\prime} P^{\prime},[\ldots]$ paralelas al eje de las $v$, satisfacen todos á la ecuación; porque, siendo $x$ cualquiera abscisa $O P$, y $v$ su correspondiente ordenada $P M$, siempre se verifica. De modo que $v=a x$ es la relación general de abscisa y ordenada, pertenecientes á cualquiera punto de la recta $O M$ que pasa por el oríjen. ${ }^{11}$

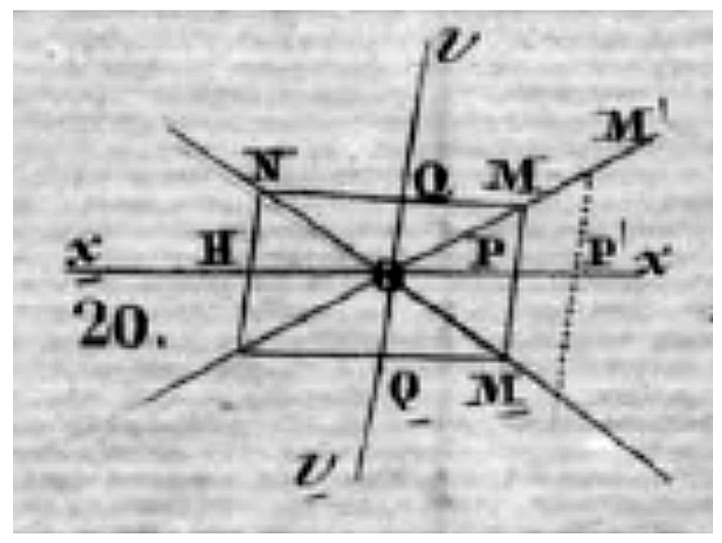

Ilustración 13. Figura 20. Odriozola, 1829.

\footnotetext{
70 Odriozola, Curso Completo de Matemáticas Puras, tomo III, 204.

${ }^{71}$ Odriozola, Curso Completo de Matemáticas Puras, tomo III, 204.
} 
Para el caso en que los ejes sean oblicuos determina la relación entre el coeficiente de la recta que pasa por el origen y el ángulo que forman los ejes: «Para mejor concebir la significación del coeficiente $a$, obsérvese que el triángulo $O M P$ da $a=\frac{B}{A}=\frac{\operatorname{sen} M O x}{\operatorname{sen} M O v} » \cdot{ }^{72}$ Lo que le lleva a considerar que en el caso de ejes perpendiculares la expresión de $a$ es más simple: "y como en el sistema perpendicular es senMOv=cosMOx, y de resultas $a=\operatorname{tang} M O x$; por esta simplicidad, siempre que fuere arbitrario el sistema de los ejes, conviene para las construcciones elegir el perpendicular». ${ }^{73}$

En el caso en que la pendiente es negativa:

Si $a$ es negativa, proviene del signo de uno de los senos, ó lo que es lo mismo de sus proporcionales $B$ y $A$. Suponiendo negativa $B$, si se baja la ordenada $P \underline{M}=-B$, y se dirige la recta $O \underline{M}$, todos los puntos de ésta satisfacen á la ecuación $v=-a x=\frac{-\bar{B}}{A} x$; pues, verifícase la proporcionalidad entre las ordenadas $v$ negativas y las abscisas $x$ positivas.

Siendo $a$ negativa por $A$, la ecuación $v=-a x=\frac{B}{-A} x$ exige que se tome $O H=-A$; y levantada $H N=B$, será $O N$ la línea, y prolongación de $O \underline{M}$. La recta $N \underline{M}$ será pues á quien corresponda la ecuación $v=-a x$, en que - $a$ espresa la razón entre los senos de los ángulos que forma con los ejes. ${ }^{74}$

Obsérvese la notación utilizada: Como la pendiente es negativa la escribe con su signo, $-a$; es decir, identifica la constante con su valor absoluto. Por otra parte, observamos que para denotar un punto con una de sus coordenadas negativas escribe el signo debajo de la letra que lo designa, por ejemplo $N \underline{M}$, de forma similar a como hace con los ejes.

En cuanto a la ecuación de la recta $v=a x+b$, hace la distinción entre constantes positivas y negativas y deduce las ecuaciones de los ejes de coordenadas y de las rectas paralelas a ellos ${ }^{75}$. También obtiene la ecuación

\footnotetext{
72 Odriozola, Curso Completo de Matemáticas Puras, tomo III, 204.

73 Odriozola, Curso Completo de Matemáticas Puras, tomo III, 204.

${ }^{74}$ Odriozola, Curso Completo de Matemáticas Puras, tomo III, 205.

75 Odriozola, Curso Completo de Matemáticas Puras, tomo III, 205-206.
} 
punto-pendiente, $v-\beta=a(x-\alpha)$, aunque él no le da ningún nombre, y la recta que pasa por dos puntos dados, $M(\alpha, \beta) y N\left(\alpha^{\prime}, \beta^{\prime}\right)$ para los que se obtienen las ecuaciones $v=\frac{\beta-\beta^{\prime}}{\alpha-\alpha^{\prime}} x+\frac{\alpha \beta^{\prime}-\alpha \prime \beta}{\alpha-\alpha^{\prime}}$ y $v-\beta=\frac{\beta-\beta^{\prime}}{\alpha-\alpha^{\prime}}(x-\alpha) \cdot{ }^{76}$

Además de las ecuaciones de la recta, también realiza manipulaciones algebraicas para determinar el punto de corte de dos rectas, el ángulo que forman, la ecuación de la bisectriz de dicho ángulo, las condiciones de paralelismo y perpendicularidad y la distancia entre dos puntos y de un punto a una recta, que plantea en general como problemas. Sin embargo, no propone problemas geométricos más complejos para resolver utilizando este método. Incluimos, por tanto, el cálculo de la bisectriz de un ángulo como ejemplo: «V. ${ }^{\circ}$ Dividir en dos partes iguales el ángulo $B O D$ formado por las rectas $O B, O D$, dadas por sus ecuaciones respectivas $v=a x, \downarrow=d x »$.

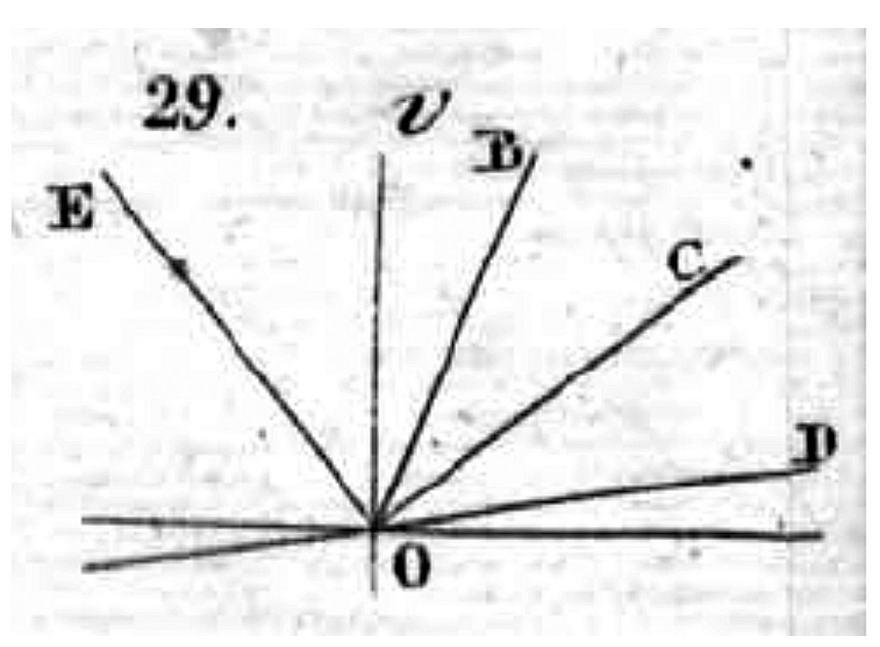

Ilustración 14. Figura 29. Odriozola, 1829.

Para resolverlo utiliza que las tangentes de los ángulos $B O C, C O D$ (fig. 29) que valen $\frac{a-\breve{a}}{1+a \breve{a}}, \frac{\breve{a}-\bar{a}}{1+\breve{a} \bar{a}}$ respectivamente, deben ser iguales. De esa ecuación obtiene otra de segundo grado, $\breve{a}^{2}-2\left(\frac{a \bar{a}-1}{a+\bar{a}}\right) \check{a}-1=0$, que

76 Odriozola, Curso Completo de Matemáticas Puras, tomo III, 210. 
no resuelve; simplemente deja planteada la solución final del problema: "Hallando el valor de $\vec{a}$ con doble signo, y sustituido en la ecuacion $\breve{v}=\breve{a} \breve{x}$, se podrán construir las rectas $O C, O E$ que correspondan á ella». ${ }^{77}$

Lo que sí hace es deducir que las dos rectas que se obtienen son perpendiculares:

Con objeto de averiguar una circunstancia notable de esta cuestion, supongamos $p$ y $q$ los dos valores de $\breve{a}$; como el último término de la ecuación de segundo grado es producto de sus raices, y en el caso actual $p q=-1$, resulta $q=-\frac{1}{p}$ de modo que las rectas $O C, O E$ de las ecuaciones $\check{v}=p \check{x}, \check{v}=-\frac{1}{p} \check{x}$ son perpendiculares entre sí. ${ }^{78}$

Obsérvese la notación utilizada por Odriozola, que no hemos observado en otros autores de la época, ${ }^{79}$ y que él mismo explica antes de comenzar con la exposición de la teoría:

37. También se determinan las constantes de la ecuación ((6)) por otro sistema de condiciones. Mas, antes de principiar la teoría debemos advertir, que muchas veces en adelante versa el cálculo sobre las circunstancias de dos ó mas líneas que se refieren á un mismo sistema de coordenadas, y para distinguir sus ecuaciones marcaremos con el acento - las letras de una de ellas, y con el acento $\smile$ las de otra cuando haya tres ecuaciones. Asimismo téngase entendido que, mientras no se diga lo contrario, se usará el sistema de coordenadas perpendiculares. ${ }^{80}$

\section{CONCLUSIONES}

Tenemos una obra dividida en dos partes claramente diferenciadas, como hemos visto, en metodología y contenidos, hecho que también se

\footnotetext{
77 Odriozola, Curso Completo de Matemáticas Puras, tomo III, 216.

78 Odriozola, Curso Completo de Matemáticas Puras, tomo III, 217.

79 Sánchez, «La Geometría Analítica en los libros de texto para secundaria y universidad en España en el siglo XIX».

80 Odriozola, Curso Completo de Matemáticas Puras, tomo III, 211.
} 
da en otros autores del este siglo XIX. Pero este texto tiene una serie de características que lo diferencian de los demás.

En primer lugar, llama la atención la notación utilizada, diferente en muchos aspectos a la de la actualidad, pero también a la utilizada por otros autores contemporáneos de Odriozola.

Por otra parte, el uso del segmento unidad de forma explícita; no solo de forma teórica sino de forma explícita en algunos casos particulares, como hemos visto.

Un elemento importante en el estudio de las cantidades negativas es que este autor estudia cómo se pasa de una cantidad positiva a negativa, y viceversa, llegando a la conclusión de que esto puede ocurrir a través de cero o infinito. Esto solo lo hacen de forma análoga Alberto Lista y Zorraquín, aunque estos estudian las magnitudes negativas de manera más formal y exhaustiva que Odriozola, especialmente Zorraquín.

También habría que señalar la forma en la que resuelve los problemas, única en este autor, mostrando en primer lugar la solución obtenida utilizando únicamente la Geometría elemental, y después la obtenida con ayuda del Álgebra. Esta manera de enfocar la solución de un problema permite dos cosas: por una parte, comparar cuál de los dos métodos da una solución más sencilla y, por otra, observar que, generalmente, la construcción geométrica de la solución algebraica es muy similar a la hecha de forma puramente geométrica; es decir, en este caso la Geometría va en auxilio del Álgebra, y además podemos deducir por qué razón el autor lleva a cabo esa construcción y no otra, cosa que no ocurre en otros autores.

Por último, esta obra aunque, según lo expuesto por el mismo autor en el prólogo, iba destinada fundamentalmente a las escuelas militares, también se utilizó como texto en los centros de enseñanza como acredita su aparición en las listas de libros de texto oficiales.

\section{Nota sobre las autoras}

Isabel María Sánchez Sierra es profesora de Educación Secundaria Obligatoria y Bachillerato. También es doctora por la Universidad de Salamanca desde el año 2015. Su línea de investigación se ha centrado en la 
Historia de la Educación Matemática, concretamente en lo que se refiere a la enseñanza de la Geometría Analítica durante el siglo XIX sobre la que realizó su tesis doctoral. Ha publicado diversos artículos sobre esta temática en revistas nacionales e internacionales e impartido conferencias en el ámbito internacional. Además, también ha publicado artículos de divulgación sobre algunos aspectos de la enseñanza de las matemáticas.

María Teresa González Astudillo es profesora titular de la Universidad de Salamanca en el área de Didáctica de la Matemática. Premio extraordinario de doctorado por dicha universidad en el año 2002. Entre sus líneas de investigación se encuentra la de Historia de la Educación en la que realizó su tesis doctoral que versaba sobre el análisis de libros de texto de Análisis Matemático desde el siglo XVIII. Ha publicado numerosos artículos e impartido conferencias en Congresos Nacionales e Internacionales, así como dirigido varias tesis doctorales en esta línea de investigación. Actualmente es la Presidenta de la Sociedad Española de Investigación en Educación Matemática que engloba a todos los investigadores del área de Didáctica de la matemática en España. Es la investigadora principal del grupo de investigación de Matemática Educativa en la Universidad de Salamanca.

\section{REFERENCIAS}

Descartes, René. La Geometría, 1637. Buenos Aires: Espasa-Calpe, 1947. Traducido por Pedro Rossell.

Enciclopedia Universal Ilustrada Europeo-Americana. «Odriozola». Madrid: Espasa-Calpe, S.A., 1929. T. 39.

Maz, Alexander. «Los números negativos en España en los siglos XVIII y XIX». PhD diss., Universidad de Granada, 2005.

Odriozola, José. Curso Completo de Matemáticas Puras. Madrid: Imprenta que fue de García, 1827-1829.

Sánchez Sierra, Isabel M. «La Geometría Analítica en los libros de texto para secundaria y universidad en España en el siglo XIX». PhD diss., Universidad de Salamanca, 2015.

Sánchez Sierra, Isabel $\mathrm{M}^{\mathrm{a}}$., y $\mathrm{M}^{\mathrm{a}}$. Teresa González Astudillo. «La Geometría Analítico-Descriptiva de Mariano Zorraquín». HISTEMAT 2, no. 3 (2016): 200-228. 
Sánchez Sierra, Isabel $\mathrm{M}^{\mathrm{a}}$., y $\mathrm{M}^{\mathrm{a}}$. Teresa González Astudillo. «La geometría analítica de Alberto Lista». Épsilon - Revista de Educación Matemática 34, no. 95 (2017): 7-24.

Sánchez Sierra, Isabel Ma $\mathrm{M}^{\mathrm{a}}$. $\mathrm{M}^{\mathrm{a}}$. Teresa González Astudillo. «La geometría analítica en España durante el siglo XIX: estudio de las soluciones negativas de una ecuación». Enseñanza de las ciencias 35, no. 3 (2017): 89-106. https:// doi.org/10.5565/rev/ensciencias.2348.

Smith, David y Marcia Latham. The Geometry of René Descartes: Translated from de French and Latin, with a facsimil of the first edition, 1637. Illinois: Open Court, 1952.

Vea, Fernando. Las matemáticas en la enseñanza secundaria en España en el siglo XIX. Zaragoza: Seminario de Historia de la Ciencia y de la Técnica de Aragón. Facultad de Ciencias (Matemáticas), 1995. 